\title{
THE THEOREMS OF OSCILLATION OF STURM AND KLEIN. (THIRD PAPER.)
}

BY PROFESSOR MAXIME BÔCHER.

(Read before the American Mathematical Society at its Fifth Summer Meeting, Boston, Mass., August 19, 1898.)

THE following pages form a continuation of two papers presented under the same title to the Society and printed on pp. 295-313 and 365-376 of the preceding volume of the Bulletin. These papers will be referred to as $T h$. of Osc. 1 and 2 respectively.

The object of the present paper is to extend the results so far established to some cases in which the coefficients of the differential equation in question are no longer continuous throughout the intervals with which we are concerned. Such extensions are made in $\S \S 2$ and 3 of the present paper but for this purpose it is necessary in $\S 1$ to establish some fundamental theorems concerning linear differential equations of the second order with discontinuous coefficients, results which are perhaps of some interest apart from the special applications here made of them. Before entering on these questions, however, it will be convenient to describe accurately the kind of discontinuities with which we shall deal, and to establish certain general theorems.

All the functions with which we shall have to deal in the present paper are, throughout the interval in which we consider them, single valued real functions of one or more real variables. Taking first the case of a function of a single variable $f(x)$, we shall consider only the case in which this function has in an interval $a \leqq x \leqq b$ a finite number of points of discontinuity.

The simplest discontinuities from some points of view are the so-called finite discontinuities* of $f(x), i$. e., discontinuities $x=c$ for which a positive quantity $M$ can be found such that in the neighborhood of $c,|f(x)|<M$. Going beyond these finite discontinuities we have discontinuities at

* Strictly speaking this includes the case in which the discontinuity is simply due to the fact that the function has not been defined at the point in question ; for example $e^{-1 / x^{2}}$ at the origin. We will, however, here once for all make the convention that in such cases we will regard the function as being so defined at the point in question as to preserve the continuity if possible. 
which the function becomes infinite for some or for all methods of approach. For our purpose we need merely to restrict these discontinuities by requiring that the functions do not become infinite more strongly than $(x-c)^{-i}$ where $0<i<1$. We may then describe the discontinuities with which we have to deal, including the finite discontinuities, as follows :

(A) The functions $f(x)$ with which we shall have to deal are such that a function $\psi(x)$ exists single valued and continuous throughout the interval $a \leqq x \leqq b$ and such that at every point of this interval except $c_{1}, c_{2}, \cdots, c_{n}$

$$
\psi(x)=\left[\left(x-c_{1}\right)\left(x-c_{2}\right) \cdots\left(x-c_{n}\right)\right]^{i} f(x),
$$

where $0<i<1$.

It should be noticed that for functions of this sort $\int f(x) d x$ extended over a part or the whole of the interval $a b$ is absolutely convergent, $i$. e., $\int|f(x)| d x$ is convergent.

We shall also have to deal with functions of two or more independent variables $x, \lambda, \mu, \cdots$ which we can describe as follows :

(B) The functions $f(x ; \lambda, \mu, \cdots)$ with which we shall have to deal are such that a function $\psi$ can be found which is a single valued and continuous function of $(x, \lambda, \mu, \cdots)$ when $a \leqq x \leqq b$, $\lambda_{2} \leqq \lambda \leqq \lambda_{1}, \mu_{2} \leqq \mu \leqq \mu_{1}, \cdots$ and such that for all of these values except when $x=c_{1}, c_{2}, \cdots, c_{n}$

$$
\psi(x ; \lambda, \mu, \cdots)=\left[\left(x-c_{1}\right)\left(x-c_{2}\right) \cdots\left(x-c_{n}\right)\right]^{i} f(x ; \lambda, \mu, \cdots)
$$

where $i$ is a constant satisfying the inequality $0<i<1$.

We give now an important theorem concerning such functions :

(C) If $f(x ; \lambda, \mu, \cdots)$ is a function of the kind described in (B) and if $x_{0}$ is a constant satisfying the inequality $a \leqq x_{0} \leqq b$, then

$$
F(x ; \lambda, \mu, \cdots)=\int_{x_{0}}^{x} f(x ; \lambda, \mu, \cdots) d x
$$

is a continuous function of $(x, \lambda, \mu, \cdots)$ for all the values in question.

In order to prove this we have merely to show that as $\Delta x, \Delta \lambda, \Delta \mu, \cdots$ approach zero independently of each other the difference :

$$
\Delta F=F(x+\Delta x ; \lambda+\Delta \lambda, \mu+\Delta \mu, \cdots)-F(x ; \lambda, \mu, \cdots)
$$


approaches zero. Now we can write

$$
\begin{aligned}
\Delta F= & \int_{x_{0}}^{x+\Delta x} f(x ; \lambda+\Delta \lambda, \mu+\Delta \mu, \cdots) d x-\int_{x_{0}}^{x+\Delta x} f(x ; \lambda, \mu, \cdots) d x \\
& +\int_{x_{0}}^{x+\Delta x} f(x ; \lambda, \mu, \cdots) d x-\int_{x_{0}}^{x} f(x ; \lambda, \mu, \cdots) d x .
\end{aligned}
$$

We have, therefore, the inequality

$$
\begin{gathered}
\Delta F\left|\leqq \int_{x_{0}}^{x+\Delta x}\right| f(x ; \lambda+\Delta \lambda, \mu+\Delta \mu, \cdots)-f(x ; \lambda, \mu, \cdots)|\cdot| d x \mid \\
+\int_{x}^{x+\Delta x}|f(x ; \lambda, \mu, \cdots)| \cdot|d x| .
\end{gathered}
$$

The second of these integrals clearly approaches zero as $\Delta x$ approaches zero. The first may be written, if we use the same notation as is used in (B), in the form

$$
\begin{aligned}
& \int_{x_{0}}^{x+\Delta n}\left|\left(x-c_{1}\right) \cdots\left(x-c_{n}\right)\right|^{-i}|\Delta \psi||d x| \\
\leqq & \int_{a}^{b}\left|\left(x-c_{1}\right) \cdots\left(x-c_{n}\right)\right|^{-i}|\Delta \psi| d x,
\end{aligned}
$$

where $\Delta \psi^{\prime}=\psi(x ; \lambda+\Delta \lambda, \mu+\Delta \mu, \cdots)-\psi(x ; \lambda, \mu, \cdots)$.

If now we denote the greatest value of $|\Delta \psi|$ in the interval $a \leqq x \leqq b$ by $\varepsilon$ we see that the integral we are considering does not exceed

$$
\varepsilon \int_{a}^{b}\left|\left(x-c_{1}\right) \cdots\left(x-c_{n}\right)\right|^{-i} d x .
$$

But owing to the uniform continuity of $\psi, \varepsilon$ clearly approaches zero when $\Delta \lambda, \Delta \mu, \cdots$ all approach zero. Accordingly the whole integral, and therefore $\Delta F$, approaches zero, as was to be proved.

(D) If $f(x ; \lambda, \mu, \cdots)$ is a function of the kind described in (B) and $\varphi(x, \lambda, \mu, \cdots)$ is throughout the region in question a single valued continuous function of $(x, \lambda, \mu, \cdots)$, then $f . \varphi$ is a function of the kind described in (B).

The proof of this theorem follows immediately from (B).

(E) If throughout the interval $a \leqq x \leqq b, \varphi(x)$ is single valued and continuous and $\varphi(c)=0(a \leqq c \leqq b)$, then if $k>0$ and $x_{0}$ is any point of $a b$

$$
\lim _{x=c}\left[(x-c)^{k} \int_{x_{0}}^{x} \frac{\varphi(x)}{(x-c)^{k+1}} d x\right]=0 .
$$


In order to prove this let us take a point $x_{1}$ so near to $c$ that when $|x-c| \leqq\left|x_{1}-c\right|,|\varphi(x)|<k \varepsilon$. Now denoting the expression which stands above in square parentheses by $F(x)$ we have

$$
F(x)=(x-c)^{k} \int_{x_{0}}^{x_{1}} \frac{\varphi(x)}{(x-c)^{k+1}} d x+(x-c)^{k} \int_{x_{1}}^{x} \frac{\varphi(x)}{(x-c)^{k+1}} d x
$$

so that when $|x-c|<\left|x_{1}-c\right|$

$$
\begin{gathered}
|F(x)|<|x-c|^{k} \int_{x_{0}}^{x_{1}} \frac{|\varphi(x)|}{|x-c|^{k+1}|d x|} \\
+k \varepsilon|x-c|^{k} \int_{x_{1}}^{x} \frac{|d x|}{|x-c|^{k+1}} \\
=|x-c|^{k} \int_{x_{0}}^{x} \frac{|\varphi(x)|}{|x-c|^{k+1}|d x|+\varepsilon\left[1-\left(\frac{x-c}{x_{1}-c}\right)^{k}\right] .}
\end{gathered}
$$

The second of these two terms is evidently less than $\varepsilon$ while the first can be made less than $\varepsilon$ by taking $|x-c|$ sufficiently small. No matter how small $\varepsilon$ may be chosen $|F(x)|$ can be made less than $2 \varepsilon$ by taking $|x-c|$ small enough, and this proves the theorem.

$\S 1$. On Linear Differential Equations of the Second Order with Discontinuous Coefficients.

Let us consider the differential equation

$$
\frac{d^{2} y}{d x^{2}}+p(x) \frac{d y}{d x}+q(x) y=0
$$

in which throughout the interval $a \leqq x \leqq b$ the coefficients $p$ and $q$ are functions of the kind described in (A). We can, of course, not expect to find solutions $y$ which satisfy (1) at all points of $a b$. We shall therefore seek functions which, together with their first derivatives are single valued and continuous throughout $a b$ and except at the points of discontinuity $\left(x=c_{1}, c_{2}, \cdots, c_{n}\right)$ of $p$ or $q$ satisfy (1).

In order to simplify matters let us first assume that there is only one point $(x=c)$ of discontinuity in $a b$. It is at once clear from (A) that we can find a positive quantity $M$ such that at all points of the interval $a \leqq x \leqq b$ (except $c$ )

$$
|p|<M|x-c|^{-i}, \quad|q|<M|x-c|^{-i} .
$$


We will now prove that the method of successive approximations enables us to find a function $y$ which, together with its first derivative, is single valued and continuous throughout the interval $a \leqq x \leqq b$, which satisfies (1) at every point of this interval except at $c$, and which at $c$ has the arbitrarily prescribed value $\gamma$ while its derivative has the arbitrarily prescribed value $\gamma^{\prime}$.*

To prove this we will let

$$
Y_{0}=\gamma+\gamma^{\prime}(x-c)
$$

and compute the quantities $Y_{1}, Y_{2}, \cdots$ from the formulæ

$$
\begin{aligned}
Y_{n}^{\prime} & =-\int_{c}^{x}\left[p Y_{n-1}{ }^{\prime}+q Y_{n-1}\right] d x, \\
Y_{n} & =\int_{c}^{x} Y_{n}^{\prime} d x,
\end{aligned}
$$

where accents denote differentiation. It should be noticed that the functions $Y_{n}^{\prime}$ and $Y_{n}$ as thus defined are continuous throughout the interval $a b$. We wish to prove that the series

$$
Y_{0}+Y_{1}+Y_{2}+\cdots
$$

converges throughout $a b$ and represents the desired solution of (1). In order to prove this let us consider by the side of the series (3) the series formed by differentiating it term by term

$$
Y_{0}^{\prime}+Y_{1}^{\prime}+Y_{2}^{\prime}+\cdots
$$

We will begin by proving (3) and (4) uniformly convergent throughout $a b$. Let us write for brevity $|x-c|=t$. Let $l$ be a positive quantity greater than 1 and such that at all points of $a b, t<l$; and finally let $C$ be a positive quantity such that at all points of $a b$ the inequalities $\left|Y_{0}\right|<C$, $\left|Y_{0}^{\prime}.\right|<C$ hold. We will first establish the inequalities

$$
\begin{aligned}
& \left|Y_{n}^{\prime}\right|<\frac{C}{n !}\left(\frac{2 M l}{1-i} t^{1-i}\right)^{n} \\
& \left|Y_{n}\right|<\frac{C}{n !}\left(\frac{2 M l}{1-i} t^{1-i}\right)^{n} .
\end{aligned}
$$

These formulæ evidently hold when $n=0$. They will therefore be established for all values of $n$ if assuming them

* A precisely similar theorem holds for homogeneous linear differential equations of order higher than the second. The proof is essentially the same as that here given. 
to hold for a given value of $n$ we can show that they hold for the next larger value. Now we have

$$
\begin{gathered}
\left|Y^{\prime}{ }_{n+1}\right| \leqq M \int_{0}^{t} t^{-i}\left[\left|Y_{n}^{\prime}\right|+Y_{n} \mid\right] d t \\
<\frac{2 C M}{n !}\left(\frac{2 M l}{1-i}\right)^{n} ! \int_{0}^{t} t^{n-(n+1) i} d t \\
=\frac{C}{(n+1) !}\left(\frac{2 M l}{1-i}\right)^{n+1} \frac{1}{l} t^{(n+1)(1-i)} \\
\quad<\frac{C}{(n+1) !}\left(\frac{2 M l}{1-i} t^{1-i}\right)^{n+1} .
\end{gathered}
$$

Furthermore

$$
\begin{gathered}
\left|Y_{n+1}\right| \leqq \int_{0}^{t}\left|Y_{n+1}^{\prime}\right| d t<\frac{C}{(n+1) !}\left(\frac{2 M l}{1-i}\right)^{n+1} \frac{1}{l} \int_{0}^{t} t^{(n+1)(i-1)} d t \\
=\frac{C}{(n+1) ![(n+1)(1-i)+1]}\left(\frac{2 M l}{1-i}\right)^{n+1} \frac{t}{l} t^{(n+1)(1-i)} \\
<\frac{C}{(n+1) !}\left(\frac{2 M l}{1-i} t^{1-i}\right)^{n+1} .
\end{gathered}
$$

Thus formulæ (5) are established. From these formulæ we can deduce the following inequalities in which the variable $t$ no longer enters

$$
\begin{aligned}
& \left|Y_{n}^{\prime}\right|<\frac{C}{n !}\left(\frac{2 M l^{2-i}}{1-i}\right)^{n} \\
& \left|Y_{n}\right|<\frac{C}{n !}\left(\frac{2 M l^{2-i}}{1-i}\right)^{n} .
\end{aligned}
$$

From (6) it appears that each term in (3) and also each term in (4) is less than the corresponding term in the exponential series

$$
\sum_{n=0}^{n=\infty} \frac{C}{n !}\left(\frac{2 M l^{2-i}}{1-i}\right)^{n}
$$

Since this last series is a series with positive constant terms and is known to be convergent, it follows that the series (3) and (4) are both uniformly convergent throughout $a b$. Each series, therefore, represents a continuous function of $x$ and (4) represents the derivative of (3). We will denote 
the value of the series (3) by $y$. We see then that $y$ is a function which, together with its first derivative, is single valued and continuous throughout $a b$. To prove that $y$ satisfies (1) at every point of $a b$ other than $c$ let us differentiate (4) term by term

$$
-\left(p Y_{0}^{\prime}+q Y_{0}\right)-\left(p Y_{1}^{\prime}+q Y_{1}\right)-\left(p Y_{2}^{\prime}+q Y_{2}\right)-\cdots .
$$

It is clear that this same series might have been obtained by multiplying (4) by $-p$ and (3) by $-q$ and adding. It is accordingly uniformly convergent throughout any part of $a b$ which does not contain $c$, and is therefore equal to $y^{\prime \prime}$. On the other hand it is equal to $-p y^{\prime}-q y$. Therefore at every point of $a b$ other than $c, y$ satisfies (1).

Finally when $x=c$ all the terms after the first in both (3) and (4) are zero while the first reduce to $\gamma$ and $\gamma^{\prime}$ respectively. Thus our theorem is established.

We will now prove that every function $y$ which satisfies (1) at all points of the interval $a \leqq x<c$ (or of the interval $c<x \leqq b$ ) approaches, as does also its derivative $y^{\prime}$, a finite limit as $x$ approaches c. To prove this let $y_{1}$ and $y_{2}$ be two of the solutions just obtained by the method of successive approximations, chosen (and this is evidently possible) so as to be linearly independent of each other. Then $y=C_{1} y_{1}+C_{2} y_{2}$ where $C_{1}$ and $C_{2}$ are two constants. Now since $y_{1}, y_{1}^{\prime}, y_{2}, y_{2}^{\prime}$ approach finite limits as $x$ approaches $c$ it follows that the same is true of $y$ and $y^{\prime}$.

Finally, we will prove that if throughout the interval $a \leqq x \leqq b, y_{1}$ and $y_{2}$ are together with their first derivatives single valued and continuous and except when $x=c$ satisfy (1), and if $y_{1}(c)=y_{2}(c)=\gamma$ and $y_{1}^{\prime}(c)=y_{2}^{\prime}(c)=r^{\prime}$, then $y_{1}=y_{2}$ throughout the interval $a b$. For consider first the case in which $\gamma$ and $\gamma^{\prime}$ are not both zero. Then if $y_{1}$ and $y_{2}$ were not identical it is clear that they must be linearly independent. Every other solution $y$ would therefore be expressible in the form : $y=C_{1} y_{1}+C_{2} y_{2}$, so that

and

$$
y(c)=\left(C_{1}+C_{2}\right) \gamma
$$

$$
y^{\prime}(c)=\left(C_{1}+C_{2}\right) \gamma^{\prime} \text {. }
$$

We thus see that $y(c)$ and $y^{\prime}(c)$ are proportional to $\gamma$ and $\gamma^{\prime}$ whereas we have proved that they can be arbitrarily chosen. This disposes of all cases except that in which $r=r^{\prime}=0$, and here the theorem above stated will be established if we can prove that $y_{1}$ and $y_{2}$ are both identically zero. This, however, follows at once from the case already 
considered as otherwise by adding $y_{1}$ or $y_{2}$ to a solution $y_{3}$ which does not vanish (or whose derivative does not vanish) at $c$ we should get a different solution which at $c$ has the same value and the same derivative as $y_{3}$, and this we have just proved to be impossible.

The results just obtained can be at once extended to the case in which there is more than one point of discontinuity of $p$ or $q$ in the interval $a b$. For this purpose it is merely necessary to break up the interval $a b$ into shorter intervals in each of which only one point of discontinuity lies. We thus get the following theorem

I. If in the interval $a \leqq x \leqq b, p$ and $q$ are functions of the kind described in (A) there exists one and only one function $y$ which together with its first derivative is single valued and continuous throughout $a b$, satisfies (1) at every point of ab except at the points of discontinuity of $p$ or $q$, and has at an arbitrarily given point of $a b$ (which may or may not coincide with one of the discontinuities of $p$ or $q$ ) an arbitrarily given value while its derivative also has at this point an arbitrarily given value.

Finally we note that theorems (E) and (F) Th. of Osc. 1, pp. 297, 298 hold for the more general case with which we are now dealing in which $p$ and $q$ are functions of the kind described in (A). The same is true of the theorem concerning the separation of the roots of two linearly independent solutions quoted in Th. of Osc. 2 , p. 366.

In $\S 3$ we shall have to deal with the case in which in (1) the coefficient $p$ is still a function of the kind described in (A)* while $q$ has a point of discontinuity $c$ at which it becomes infinite more strongly than the functions described in (A) but only in such a way that the function $(x-c) q$ belongs to the class described in (A). We will here again begin by confining ourselves to the case in which $c$ is the only point of discontinuity of $p$ or $q$ in the interval $a \leqq x \leqq b$, and we will prove that in this case the method of successive approximations enables us to find a function $y_{1}$ which together with its first derivative is single valued and continuous throughout the interval $a \leqq x \leqq b$, which satisfied (1) at every point of this interval, except at $c$, and which vanishes at $c$ while its derivative has at $c$ the arbitrarily prescribed value $\gamma^{\prime} \cdot \dagger$

\footnotetext{
* In fact in the special case with which we there have to deal $p=0$.

†This is merely a special case of the following theorem which can be proved by the same method: If $p_{1}, p_{2}, \cdots, p_{k},(x-c) p_{k}+\overline{\overline{1}},(x-c)^{2} p_{k+2}$, $\cdots,(x-c)^{n-k} p_{n}$ are functions of the kind described in (A) whose only discontinuity is at $c$, then a function $Y$ exists and (an be found by the method above used which with its first $n-1$ derivatives is single valued
} 
In order to prove this we will take as a first approximation

$$
Y_{0}=\gamma^{\prime}(x-c),
$$

and form the functions $Y_{1}, Y_{2}, \cdots$ by means of the formulæ $(2)$, p. 26. The first of the two integrals in (2) is now not obviously convergent since $q$ has at $c$ a discontinuity up to which we cannot in general integrate. In order to prove this integral convergent it is clearly sufficient to prove that

$$
Y_{n}=(x-c) \varphi_{n}(x)
$$

where $\varphi_{n}(x)$ is continuous at $c$. This formula holds when $n=0$, for then we have $\varphi_{0}=\gamma^{\prime}$. If then, assuming that it is true for a given value of $n$, we can prove it true for the next larger value the convergence of our integral will be established. We will then assume the formula true when we give $n$ the value $n-1$. Then $Y_{n}^{\prime}$ is a continuous function of $x$ when $a \leqq x \leqq b$, and the same is therefore true of $Y_{n}$. Remembering that $Y_{n}(c)=0$ we have by the law of the mean

$$
Y_{n}(x)=(x-c) Y_{n}^{\prime}(x)
$$

where $\bar{x}-c|<| x-c \mid$. We have therefore $\varphi_{n}(x)=Y_{n}^{\prime}(\bar{x})$, and since $Y_{n}^{\prime}$ is continuous at $c$ and $Y_{n}^{\prime}(c)=0$ we see that $\varphi_{n}(x)$ approaches the limit zero as $x$ approaches $c$. If then we define $\varphi_{n}(x)$ to have the value zero when $x=c$ we see that $\varphi_{n}$ is continuous at $c$ as well as elsewhere.

Having thus justified the definition of the quantities $Y_{n}$ the next step is to prove that the series (3) and (4), p. 26 are uniformly convergent. For this purpose we need a few inequalities.

In the first place it is clear that we can find a positive quantity $M$ such that at all points of $a b$ (other than $c$ )

$$
|p|<M|x-c|^{-i}, \quad|x-c||q|<M|x-c|^{-i} .
$$

We then establish by mathematical induction (the reasoning being almost identical with that used in the analogous case on p. 27) the following inequalities

and continuous throughout $a b$, which at every point of $a b$ except $c$ satisfies the differential equation:

$$
\frac{d^{n} y}{d x^{n}}+p_{1} \frac{d^{n-1} y}{d x^{n-1}}+\cdots+p_{n} y=0
$$

and which at $c$ vanishes together with its first $n-k-1$ derivatives while the derivatives $\frac{d^{n-k} y}{d x^{n-k}}, \cdots \frac{d^{n-1} y}{d x^{n-1}}$ have arbitrarily prescribed values at $c$. 


$$
\begin{aligned}
& \left|Y_{n}^{\prime}\right|<\frac{\left|\gamma^{\prime}\right|}{n !}\left(\frac{2 M t^{1-i}}{1-i}\right)^{n} \\
& \left|Y_{n}\right|<\frac{\left|\gamma^{\prime}\right|}{n !}\left(\frac{2 M t^{1-i}}{1-i}\right)^{n} t
\end{aligned}
$$

where as before we have let $|x-c|=t$. By means of these inequalities the uniform convergence of (3) and (4) is established at once as on p. 27, and as there it is shown that (3) therefore represents a function $y_{1}$ of the kind desired. Our theorem is thus proved.

A few simple facts about these functions $y_{1}$ must be noted before we go further. In the first place it is clear that if $\gamma^{\prime}=0 \quad y_{1}$ is identically zero. Apart from this trivial case it is clear that $y_{1}$ cannot have an infinite number of roots in the interval $a b$, for these roots could (by (E) Th. of Osc. 1, p. 297) have no other point than $c$ as a limiting point, and this would clearly require the vanishing of $y_{1}^{\prime}(c)$.

In the second place we can write

$$
y_{1}(x)=(x-c) E(x),
$$

where $E(x)$ is single valued and continuous throughout ab and $E(c)=\gamma^{\prime}$. For $E(x)$ will of course be continuous everywhere in $a b$ except at $c$. In order to prove our theorem it is therefore sufficient to show that

$$
\lim _{x=c} E(x)=\gamma^{\prime} \text {. }
$$

This follows at once from the law of the mean which tells us that $y_{1}(x)=(x-c) y_{1}^{\prime}(\bar{x})$ where $|\bar{x}-c|<|x-c|$. We have then $E(x)=y_{1}^{\prime}(\bar{x})$, so that

$$
\lim _{x=c} E(x)=\lim _{\bar{x}=c} y_{1}^{\prime}(\bar{x})=\gamma^{\prime} .
$$

It is clear that the solutions $y_{1}$ so far obtained are linearly dependent, differing only in the value of the constant factor $\gamma^{\prime}$. We proceed to prove that any solution of (1) linearly independent of the solutions $y_{1}$ just obtained approaches a finite limit different from zero as $x$ approaches $c$.

In order to prove this let us remember that if $x_{0}$ is a point so near to $c$ that $y_{1} \neq 0$ when $|x-c| \leqq\left|x_{0}-c\right|$

$$
y_{2}=y_{1} \int_{x_{0}}^{x} \frac{e^{-\int_{x_{0}}^{x} p d x}}{y_{1}^{2}} d x
$$


is a solution of (1) linearly independ ent of $y_{1}$. Writing as above $y_{1}=(x-c) E(x)$ we have

$$
y_{2}=(x-c) E(x) \int_{x_{0}}^{x} \frac{e^{-\int_{x_{0}}^{x} d x}}{(x-c)^{2}[E(x)]^{2}} d x \text {. }
$$

Now we will write

$$
\frac{1}{[E(x)]^{2}} e^{-\int_{x_{0}}^{x} p d x}=K+F(x),
$$

where $K$ is a constant different from zero and $F(x)$ is single valued and continuous while $F(c)=0$. We then get

$y_{2}=-K E(x)+K E(x) \frac{x-c}{x_{0}-c}+(x-c) E(x) \int_{x_{0}}^{0 x} \frac{F(x)}{(x-c)^{2}} d x$

As $x$ approaches $c$ it is clear that the first of these three terms approaches a finite limit different from zero, while the second approaches zero. By (E) the third also approaches zero. $y_{2}$ therefore approaches a limit different from zero. Now every solution of (1) linearly independent of $y_{1}$ may be written in the form : $y=C_{1} y_{1}+C_{2} y_{2}$ where $C_{2} \neq 0$ so that the truth of our theorem is evident.*

The functions $y$, with which we have been dealing may of course be extended continuously throughout larger intervals where $p$ and $q$ have other discontinuities than $c$ provided that these discontinuities are of the kind described in (A). We are thus led to the following theorem :

II. If in the interval $a \leqq x \leqq b, p$ and $(x-c) q$ (where $a \leqq$ $c \leqq b$ )are functions of the kind described in (A), there exists one and except for a multiplicative constant only one, function $y_{1}$ which together with its first derivative is single valued and continuous throughout ab, satisfies the differential equation (1) at every point of $a b$ except at the points of discontinuity of $p$ and $q$, and vanishes when $x=c$.

We have so far regarded the coefficients $p$ and $q$ as functions of $x$ only. We shall, however, in what follows be obliged to regard them as involving also a parameter $\lambda$.

Let us first suppose that when $a \leqq x \leqq b, \lambda_{2} \leqq \lambda \leqq \lambda_{1}, p$ and $q$ are functions of the kind described in (B). We begin as before with the case in which there is only one value $x=c$ for which $p$ or $q$ is discontinuous, and apply the method of

* It may be added that the derivatives of solutions linearly indepen. dent of $y_{1}$ will not in general approach finite limits at $c$. 
successive approximations regarding $\gamma$ and $\gamma^{\prime}$ as constants.* In the analysis of pp. 26-28 we have merely to consider the nature of the dependence upon $\lambda$ of the quantities which appear. Thus $M$ may be taken as independent of $\lambda . Y_{n}$ and $Y_{n}^{\prime}$ will be by (C) continuous functions of $(x, \lambda)$. The analysis moreover proves that (3) and (4) are uniformly convergent not merely with regard to $x$ but with regard to $(x, \lambda)$ so that $y$ and $y^{\prime}$ are continuous functions of $(x, \lambda)$. This result we state as follows:

III. If when $\alpha \leqq x \leqq b$ and $\lambda_{2} \leqq \lambda \leqq \lambda_{1} p$ and $q$ are functions of the kind described in (B), the solution of (1) which together with its first derivative is constant for an arbitrarily given value $x=x_{0}\left(a \leqq x_{0} \leqq b\right)$, is together with its first derivative a continuous function of $(x, \lambda)$ when $a \leqq x \leqq b, \lambda_{2} \leqq \lambda \leqq \lambda_{1}$.

Finally we come to the case of differential equations in which $p$ and $(x-c) q$ are functions of the kind considered in (B), concerning which the following theorem may be stated :

IV. If when $a \leqq x \leqq b$ and $\lambda_{2} \leqq \lambda \leqq \lambda_{1}, p$ and $(x-c) q$ (where $a \leqq c \leqq b$ ) are functions of the kind described in (B), the solution of (1) which vanishes when $x=c$ and whose derivative has at this point $a$ constant value is when $a \leqq x \leqq b$ and $\lambda_{2} \leqq \lambda \leqq \lambda_{1}$ together with its first derivative a continuous function of $(x, \lambda)$.

The proof here is almost precisely the same as for theorem III. The only point which presents any difficulty is the proof that $Y_{n}^{\prime}$ is a continuous function of $(x, \lambda)$. In order to prove this ${ }^{n}$ let us write as on p. 30

so that

$$
Y_{n}(x, \lambda)=(x-c) \varphi_{n}(x, \lambda)
$$

$$
Y_{n}^{\prime}(x, \lambda)=-\int_{c}^{x}\left[p Y_{n-1}^{\prime}+(x-c) q \varphi_{n-1}\right] d x .
$$

If then we can prove that the functions $\varphi_{n}$ are continuous functions of $(x, \lambda)$ when $x=c$ (they are obviously continuous for other values of $x$ ) we could as before infer that the functions $Y_{n}^{\prime}$ are continuous functions of $(x, \lambda)$. We shall of course here again use the method of mathematical induction : noting that $\varphi_{0}=r^{\prime}$ is continuous, then assuming that $\varphi_{1}, \varphi_{2}, \cdots$, $\varphi_{n-1}$ are continuous. From this it follows that $Y_{n}^{\prime}$ is a continuous function of $(x, \lambda)$. Now we have (see p. 30)

$$
\varphi_{n}(x, \lambda)=Y_{n}^{\prime}(\bar{x}, \lambda)
$$

where $|\bar{x}-c|<|x-c|$, whence the continuity of $\varphi_{n}$ when $x=c$ follows at once.

\footnotetext{
* The result and the method of proof would remain the same if $\gamma$ and $\gamma^{\prime}$ were any continuous functions of $\lambda$.
} 
§2. Extension of Results of Previous Papers to Equations with Discontinuous Coefficients for which the Boundary Conditions may Remain General.

Leaving for the next section equations for which we should have to base our results on Theorems II and IV of the last section, we consider in the present section extensions of our previous results based on Theorems I and III. Here we may state at once the theorem

V. All the theorems stated in Th. of Osc. 1, \$§ 1, 2, and Th. of Osc. $2, \S 1$, remain true for differential equations whose coeffi. cients instead of being continuous functions of $x$ or $(x, \lambda)$ are functions of the kind described in (A) or (B).*

The truth of this statement is at once obvious when we refer to the proofs of the theorems in question. These proofs would require only a few modifications which, in view of the results of the last section, are so obvious that it does not seem necessary to dwell upon the matter further.

There remain then to be generalized the results contained in Th. of Osc. $2, \S 2$. We start here from the differential equation (1) in which

$$
q(x)=\chi(x)+\psi(x)\left[C_{k} x^{k}+C_{k-1} x^{k-1}+\cdots+C_{0}\right],
$$

and where $p, \chi$, and $\psi$ are independent of the parameters $C_{0}, \cdots, C_{k}$. We have to consider the $k+1$ finite intervals $a_{0} b_{0}, a_{1} b_{1}, \cdots, a_{k} b_{k}$ such that

$$
a_{0}<b_{0} \leqq a_{1}<b_{1} \leqq a_{2}<b_{2} \cdots \cdots<b_{k-1} \leqq a_{k}<b_{k},
$$

and the $k+1$ points $\xi_{0}, \xi_{1}, \cdots, \xi_{k}$ such that $\alpha_{i}<\xi_{i}<b_{i}$. We will now assume first that each of the functions

$$
t_{i}=g_{i} \int_{\xi_{i}}^{c-\iint_{p d x}^{x}} \underbrace{x}_{\xi_{i}} d x+f_{i} \quad(i=0,1, \cdots, k)
$$

(where $g_{i}$ is any constant not zero and $f_{i}$ any constant) is a continuous function of $x$ throughout the interval $a_{i} \leqq x \leqq b_{i}$. This will of course be the case if $p$ is a function of the kind described in (A), but it will also be true in some other cases which we shall find important. $\dagger$ This first assump-

* In fact, in Th. of Osc. 1, Theorem VIII, and Th. of Osc. 2, Theorem III, it is not necessary that $\phi(x, \lambda)$ should be a function of the kind described in (B) for the whole interval $L>\lambda>l$. It is sufficient that this should be the case for every interval $\lambda_{2} \leqq \lambda \leqq \lambda_{1}$ contained in the interval just mentioned.

†Cf. for instance p. 37. 
tion makes the interval $a_{i} b_{i}$ of the $x$-axis correspond in a one to one manner to a finite interval $\sigma_{i} \tau_{i}$ of the $t_{i}$ axis.

Secondly we will assume that

$$
\chi e^{2 \int_{\xi_{i}}^{x}} \text { and } \psi^{\prime} e^{2 \int_{\xi_{i}}^{x} x} \quad(i=0,1, \cdots, k)
$$

are throughout the interval $\sigma_{i} \tau_{i}$ (including the ends) functions of $t_{i}$ of the kind described in (A); and

Thirdly that $\psi(x)$ does not change sign in any one of these intervals or have an infinite number of roots in them.

Under these circumstances we have the following theorem

VI. There is one and only one real deterwination of the parameters $C_{0}, C_{1}, \cdots, C_{k}$ for which the equation (1) has $k+1$ solutions $y_{0}, y_{1}, \cdots, y_{k}$ such that $y_{i}(i=0,1, \cdots, k)$ has just $m_{i}$ roots in the interval $a_{i}<x<b_{i}$ and satisfies the conditions

$$
\begin{aligned}
& \alpha_{i}^{\prime} y_{i}\left(a_{i}\right)-\alpha_{i}\left[\frac{d y_{i}}{d t_{i}}\right]_{x=a_{i}}=0 \\
& \beta_{i}^{\prime} y_{i}\left(b_{i}\right)-\beta_{i}\left[\frac{d y_{i}}{d t_{i}}\right]_{x=b_{i}}=0 .
\end{aligned}
$$

Here the quantities $m_{i}$ are any integers positive or zero, and $\alpha_{i}, \alpha_{i}^{\prime}, \beta_{i}, \beta_{i}^{\prime}$ are any real quantities subject only to the restriction that $\alpha_{i}$ and $\alpha_{i}^{\prime}$ are not both zero and that $\beta_{i}$ and $\beta_{i}^{\prime}$ are not both zero.

The proof of this theorem is practically identical with the proof of Theorem IV., Th. of Osc. 2* so that we will omit it here. It should be noticed that this theorem includes as special cases Theorems IV and V of Th. of Osc. 2 .

An important application of Theorem VI is to the general linear differential equation of the second order which is everywhere regular

$$
\begin{gathered}
\frac{d^{2} y}{d x^{2}}+\left(\frac{1-x_{1}{ }^{\prime}-x_{1}^{\prime \prime}}{x-e_{1}}+\cdots+\frac{1-x_{n}{ }^{\prime}-x_{n}^{\prime \prime}}{x-e_{n}}\right) \frac{d y}{d x} \\
+\frac{1}{f(x)}\left[\frac{x_{1}{ }^{\prime} x_{1}^{\prime \prime} f^{\prime}\left(e_{1}\right)}{x-e_{1}}+\cdots+\frac{x_{n}{ }^{\prime} x_{n}^{\prime \prime} f^{\prime}\left(e_{n}\right)}{x-e_{n}}\right. \\
\left.+C_{n-2} x^{n-2}+C_{n-3} x^{n-3}+\cdots+C_{0}\right] y=0,
\end{gathered}
$$

where $f(x)=\left(x-e_{1}\right)\left(x-e_{2}\right) \cdots\left(x-e_{n}\right)$.

* The greatest difference comes in in (a) Th. of Osc. 2, p. 373, where we must now prove $\phi_{k}$ to be not a continuous function of $\left(t_{k}, C_{k}\right)$ but a function of the kind described in (B). 
At this point we will define a term which we shall find it convenient to make use of in what follows. By the fundamental solutions of (7) corresponding to the singular point $e_{i}$ we shall understand the solutions of (7) which can be developed about $e_{i}$ in the form*

$$
\begin{gathered}
y^{(1)}=\left(x-e_{i}\right)^{\kappa_{i}}\left[1+g_{1}{ }^{\prime}\left(x-e_{i}\right)+g_{2}{ }^{\prime}\left(x-e_{i}\right)^{2}+\cdots\right], \\
y^{(2)}=\left(x-e_{i}\right)^{\kappa_{i}{ }^{\prime \prime}}\left[1+g_{1}^{\prime \prime}\left(x-e_{i}\right)+g_{2}^{\prime \prime}\left(x-e_{i}\right)^{2}+\cdots\right] .
\end{gathered}
$$

If we have occasion to distinguish between these two solutions we shall do so by speaking of the first as corresponding to the exponent $x_{i}^{\prime}$ the second to $x_{i}^{\prime \prime}$. Moreover if we recall that a non-singular point $c$ of $(7)$ may be regarded as having exponents 1 and 0 we see that it will be natural to understand by the fundamental solutions of (7) corresponding to a non-singular point $c$ the solutions which can be developed about $c$ in the forms

$$
\begin{aligned}
& y^{(1)}=(x-c)+g_{2}{ }^{\prime}(x-c)^{2}+g_{3}{ }^{\prime}(x-c)^{3}+\cdots, \\
& y^{(2)}=1 \quad+g_{2}{ }^{\prime \prime}(x-c)^{2}+g_{3}{ }^{\prime \prime}(x-c)^{3}+\cdots .
\end{aligned}
$$

We assume as before that the coefficients of $(7)$ are real for real values of $x$ (Cf. Th. of Osc. 2, p. $375(a)-(d)$ ); and letting $k \leqq n-2$ we consider the $k+1$ intervals $a_{i} \leqq x \leqq b_{i}(i=0,1, \cdots, k)$ where

$$
a_{0}<b_{0} \leqq a_{1}<b_{1} \leqq a_{2}<b_{2} \cdots \cdots<b_{k-1} \leqq a_{k}<b_{k} \text {. }
$$

If no singular point lies in any of these intervals it is clear as stated in Th. of Osc. 2, p. 375, that the simple form of Klein's theorem of oscillation is at once applicable. Let us now enquire how it is if one of the intervals $a_{i} b_{i}$ just reaches up with one of its ends to one of the real singular points of $(7)$. Let us say for the sake of distinctness $a_{i}=e_{1}$. There are two cases to consider :

1. The case in which the exponents $x_{1}^{\prime}, x_{1}{ }^{\prime \prime}$ are conjugate imaginaries. Here it is at once obvious from the form of development about $e_{1}$ that every real solution of (7) has an infinite number of roots in the neighborhood of $e_{1}$ so that the theorem of oscillation cannot be extended to this case. We need then merely to consider :

2. The case in which $x_{1}^{\prime}, x_{1}^{\prime \prime}$ are real. We will at first consider here only the special case (to which, however, as

* In case $\kappa_{i}^{\prime}-\kappa_{i}^{\prime \prime}$ is an integer, that one of these series into which the smaller of the exponents enters would have to be replaced by a more complicated expression which, however, we shall not have occasion to use. 
we shall see presently the general case can be reduced*) in which $x_{1}^{\prime \prime}=0$ while $x_{1}{ }^{\prime}>0$. Here

$$
\begin{aligned}
t_{i} & =g_{i} \int_{\xi_{i}}^{x} e^{-\int_{\xi_{i}}^{x}\left[\frac{1-\kappa_{1}^{\prime}}{x-e 1}+\frac{1-\kappa_{2}{ }^{\prime}-\kappa_{2}^{\prime \prime}}{x-e_{2}}+\ldots+\frac{1-\kappa_{n^{\prime}}-\kappa_{n^{\prime \prime}}}{x-e_{n}}\right] d x} d x+f_{i} \\
& =\left(x-e_{1}\right)^{\kappa_{1}^{\prime}} E(x)+\sigma_{i},
\end{aligned}
$$

where $E(x)$ is continuous throughout the interval $e_{1} \leqq x \leqq b_{i}$ and $E\left(e_{1}\right) \neq 0$. The condition of continuity of $t_{i}$ is therefore satisfied since by hypothesis $x_{1}^{\prime}>0$.

A relation which we shall need in a moment can at once be deduced from the value of $t_{i}$ just obtained, viz :

$$
x-e_{1}=\left(t_{i}-\sigma_{i}\right)^{\frac{1}{\kappa_{1}{ }^{\prime}}} E_{1}\left(t_{i}\right)
$$

where $E_{1}$ is a single valued and continuous function of $t_{i}$ throughout the interval $\sigma_{i} \tau_{i}$ including the ends, and

$$
E_{1}\left(\sigma_{i}\right) \neq 0 \text {. }
$$

Going a step further we have

$$
\begin{gathered}
\chi(x)=\frac{1}{f(x)}\left[\frac{x_{2}{ }^{\prime} x_{2}{ }^{\prime \prime} f^{\prime}\left(e_{2}\right)}{x-e_{2}}+\cdots+\frac{x_{n}{ }^{\prime} x_{n}{ }^{\prime \prime} f^{\prime}\left(e_{n}\right)}{x-e_{n}}\right. \\
\left.+C_{n-2} x^{n-2}+\cdots+C_{k+1} x^{k+1}\right], \\
\psi(x)=\frac{1}{f(x)},
\end{gathered}
$$

where $f(x)=\left(x-e_{1}\right)\left(x-e_{2}\right) \cdots\left(x-e_{n}\right)$, and finally $e^{2 \int_{\xi_{i}}^{x}{ }^{p d x}}=k\left(x-e_{1}\right)^{2-2 \kappa_{1}^{\prime}}\left(x-e_{2}\right)^{2-2 \kappa_{2}^{\prime}-2 \kappa_{2}{ }^{\prime \prime}} \cdots\left(x-e_{n}\right)^{2-2 \kappa_{n}{ }^{\prime}-2 \kappa_{n}^{\prime \prime}}$

where $k$ is a constant different from zero. We may therefore write, using the value of $x-e_{1}$ in terms of $t_{i}$ above obtained

$$
\begin{aligned}
& \chi(x) e^{2 \int_{\xi_{i}}^{x} d x}=\left(x-e_{1}\right)^{1-2 \kappa_{1^{\prime}}} F_{1}(x)=\left(t_{i}-\sigma_{i}\right)^{\frac{1}{\kappa_{1}^{\prime}}-2} \Phi_{1}\left(t_{i}\right), \\
& \psi(x) e^{2 \int_{\xi i}^{x} d x}=\left(x-e_{1}\right)^{1-2 \kappa^{\prime}} F_{2}(x)=\left(t_{i}-\sigma_{i}\right)^{\frac{1}{\kappa_{1}}{ }^{\prime}}{ }^{-2} \Phi_{2}\left(t_{i}\right),
\end{aligned}
$$

* Except the case of equal exponents which as we shall see is excluded. 
where $F_{1}$ and $F_{2}$ are both continuous throughout the interval $e_{1} \leqq x \leqq b_{i}$, and $\Phi_{1}$ and $\Phi_{2}$ are both continuous throughout the interval $\sigma_{i} \tau_{i}$ including the ends. The functions

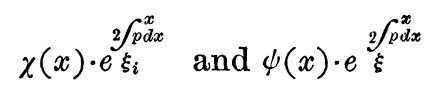

are therefore functions of $t_{i}$ of the kind described in (A) provided that $0<x_{1}^{\prime}<1$. If this last inequality holds theorem VI may therefore be applied. Moreover it is evident that instead of allowing one of the points $a_{0}, b_{0}, a_{1}, b_{1}, \cdots$ to coincide with a singular point of (7) we might have allowed any number of them to do so provided that one of the exponents of each of the singular points thus involved is zero while the other is real, greater than zero, and less than one.

The conditions to which the solutions $y_{i}$ are subjected at the points $a_{i} b_{i}$ may be stated in the cases with which we are now concerned in a very simple form if we use the conception of the fundamental solutions corresponding to these points. Suppose for instance that the singular point $e_{1}$ is one of the points $a_{i}$, then $y_{i}$ satisfies the condition

$$
\alpha_{i}^{\prime} y_{i}\left(e_{1}\right)-\alpha_{i}\left(\frac{d y_{i}}{d t_{i}}\right)_{x=e_{1}}=0 .
$$

Now $y_{i}$ can be written $k^{(1)} y^{(1)}+k^{(2)} y^{(2)}$ where $k^{(1)}$ and $k^{(2)}$ are constants and $y^{(1)}$ and $y^{(2)}$ are the fundamental solutions corresponding to the exponents $x_{1}^{\prime}$ and 0 respectively at $e_{1}$. Substituting this value of $y_{i}$ into the above condition we readily see, when we use the explicit expression for $t_{i}$ in terms of $x$, that the condition reduces to

$$
\alpha_{i}^{\prime} k^{(2)}-\alpha_{i} K k^{(1)}=0
$$

where $K$ is a constant different from zero and independent of the parameters $C$. Now since $\alpha_{i}^{\prime}$ and $\alpha_{i}$ can be chosen as any two constants not both zero we see at once that the condition can be expressed by saying that $y_{i}$ is to be proportional to an arbitrarily chosen linear combination of the fundamental solutions corresponding to $e_{1}$.

The condition $\gamma^{\prime} y(c)-\gamma y^{\prime}(c)=0$ at a non-singular point may also be stated in this form, for letting $y=k^{(1)} y^{(1)}+$ $k^{(2)} y^{(2)}$, where $y^{(1)}$ and $y^{(2)}$ are the fundamental solutions corresponding to $c$, we see at once that our condition reduces to $\gamma^{\prime} k^{(2)}-\gamma k^{(1)}=0$, and this is equivalent to requiring that $y$ be proportional to an arbitrarily chosen linear combination of $y^{(1)}$ and $y^{(2)}$. 
So far we have merely considered the case in which one of the exponents of each of the singular points with which we have to deal is zero. Let us now consider the more general case in which the exponents $x_{i}^{\prime}$ and $x_{i}^{\prime \prime}$ of these points are any real quantities where $x_{i}^{\prime}>x_{i}^{\prime \prime}$. We will let $x_{i}^{\prime}-x_{i}^{\prime \prime}=x_{i} ;$ and denote by $\Pi\left(x-e_{i}\right)^{\kappa_{i}{ }^{\prime \prime}}$ the product of the expressions $\left(x-e_{i}\right)^{\kappa_{i}{ }^{\prime \prime}}$ taken for all the singular points which coincide with any of the points $a_{i}$ or $b_{i}$. Now let

$$
y=\Pi\left(x-e_{i}\right)^{\kappa_{i}{ }^{\prime \prime} \cdot \bar{y}}
$$

and we get as the differential equation satisfied by $\bar{y}$ an equation differing from (7) in only two respects :

1st. the exponents of the points $e_{i}$ which coincide with points $a_{i}$ or $b_{i}$ are now $x_{i}$ and 0 instead of $x_{i}^{\prime}$ and $x_{i}^{\prime \prime}$.

$2 \mathrm{~d}$, the parameters $\bar{C}_{0}, \bar{C}_{1}, \cdots \bar{C}_{n-2}$ of the transformed equation are connected with the parameters $C_{0}, C_{1}, \cdots, C_{n-2}$ of the original equation by relations of the form : $C_{i}=C_{i}+k_{i}$ where the constants $k_{i}$ are independent of the $C^{\prime}$ s.

We may therefore determine the parameter $\bar{C}$ by applying the theorem of oscillation to the transformed equation provided that $0<x_{i}<1$; and the $\bar{C}$ 's being thus determined the $C^{\prime}$ s are uniquely determined. It remains then merely to show that we can so arrange the conditions of the theorem of oscillation when we apply it to the transformed equation as to give any desired conditions for the original equation.

In the first place it is clear that the roots of a solution $y_{i}$ in an interval $a_{i}<x<b_{i}$ are precisely equal to those of the corresponding solution $\bar{y}_{i}$ in this interval, and therefore in particular that $y_{i}$ and $\bar{y}_{i}$ have the same number of roots in this interval. Moreover if $y^{(1)}$ and $y^{(2)}$ are the fundamental solutions of (7) corresponding to a point $c$ (whether this point be singular or non-singular is immaterial) $\bar{y}^{(1)}$ and $\bar{y}^{(2)}$ although not themselves the fundamental solutions of the transformed equation differ from them only by the same constant factor. We may then state the following theorem :

VII. If the coefficients of equation (7) are real for real values of $x$ and if the $k+1$ intervals $(k \leqq n-2) a_{0} b_{0}, a_{1} b_{1}, \cdots, a_{k} b_{k}$ are so situated that

$$
\text { 1st, } a_{\theta}<b_{0} \leqq a_{1}<b_{1} \leqq a_{2}<b_{2} \cdots \cdots<b_{k-1} \leqq a_{k}<b_{k} ;
$$

$2 \mathrm{~d}$, no singular point of (7) lies in any of the intervals

$$
a_{i}<x<b_{i}(i=0,1, \cdots, k) \text {; }
$$


$3 \mathrm{~d}$, the points $a_{i}$ and $b_{i}$ themselves, so far as they are singular points of (7), have unequal real exponents whose difference is numerically less than 1 ;

Then, the quantities $m_{i}$ being any integers positive or zero, there is one and only one real determination of the parameters $C_{0}, C_{1}, \cdots, C_{k}$ for which (7) has $k+1$ solutions $y_{0}, y_{1}, \cdots, y_{k}$ such that $y_{i}$ has just $m_{i}$ roots in the interval $\alpha_{i}<x<b_{i}$ and at each extremity of this interval is proportional to an arbitrarily chosen linear combination of the fundamental solutions corresponding to this extremity.

§3. Extension of Results of Previous Papers to Equations with Discontinuous Coefficients for which the Boundary Conditions must be Specialized.

We will begin here by considering equations of the form :

$$
\frac{d^{2} y}{d x^{2}}=\varphi \cdot y
$$

where the coefficient $\varphi$ is such that throughout the interval $a \leqq x \leqq b,(x-a) \varphi$ is a function of the kind described in (A) or (B). Here we see by Theorems II and IV that we can no longer require of solutions $y$ that they shall satisfy the conditions $y(a)=\alpha, y^{\prime}(a)=\alpha^{\prime}$ where $\alpha$ and $\alpha^{\prime}$ are arbitrarily given, but that we must now restrict ourselves to the boundary condition $y(a)=0$. With this restriction, however, many of the results of the previous papers may be extended to the case now before us by methods practically identical with those there given. We may say :

VIII. All the theorems contained in Th. of Osc. 1, $\$ 1,2$ as well as Theorem III, Th. of Osc. 2 remain true if instead of $\varphi$ being a continuous function of $x$ or $(x, \lambda),(x-\alpha) \varphi$ is a function of the kind described in (A) or (B), provided we let $\alpha=0$ in these theorems.

Theorems almost identical with these would of course hold if the points $a$ and $b$ were interchanged (Cf. Th. of Osc. 1, §2, last paragraph).

'The methods used in the previous papers are not, however, applicable to the case in which $\varphi$ becomes infinite both at $a$ and at $b$ more strongly than the functions described in (A) or (B). We shall, therefore, need a somewhat new method to prove the following theorem of oscillation :

IX. If when $a \leqq x \leqq b$ and $L>\lambda>l, *(x-a)(x-b) \varphi(x, \lambda)$ is a function of the kind described in (B), and if when $a \leqq x \leqq b$

$*$ We may have $L=+\infty$ or $l=-\infty$ or both $L$ and $l$ may be infinite. Cf. also the first footnote on p. 34 which applies here. 
and $L>\lambda^{\prime}>\lambda^{\prime \prime}>l, \varphi\left(x, \lambda^{\prime}\right) \geqq \varphi\left(x, \lambda^{\prime \prime}\right) ; \dagger$ if, moreover

$$
\lim _{\lambda=L} \varphi(x, \lambda)=+\infty \text { and } \lim _{\lambda=l} \varphi(x, \lambda)=-\infty
$$

except perhaps for a finite number of values of $x$ in the interval $a \leqq x \leqq b$; then there will be one and only one value of $\lambda$ in the interval $L>\lambda>l$ for which (8) has a solution which has just $m$ roots ( $m$ being any integer positive or zero) in the interval $a<x<b$ and which vanishes when $x=a$ and when $x=b$.

In order to prove this let us take a point $c$ so that $a<c<b$. Let us denote by $y_{1}$ and $y_{2}$ solutions of (8) which vanish at $a$ and $b$ respectively. It will be convenient to speak in the following proof of the aggregate number of roots of $y_{1}$ and $y_{2}$ in $a b$ meaning thereby the integer obtained by adding to the number of roots of $y_{1}$ in the interval $a<x<c$ the number of roots of $y_{2}$ in the interval $c<x<b$ and increasing this number by 1 if $y_{1}$ or $y_{2}$ (or both) vanish at $c$. Our theorem will clearly be proved if we can show that there is one and only one value of $\lambda$ in the interval $L>\lambda>l$ for which $y_{1}$ and $y_{2}$ are linearly dependent while this aggregate is $m$. For if $y_{1}$ and $y_{2}$ are linearly dependent they can be made equal by multiplying one of them by a suitable constant.

Now we see from VIII (and Th. of Osc. 2, theorem III) that there is one and only one value of $\lambda$ in the interval $L>\lambda>l$, say $\lambda_{m_{1}}{ }^{\prime}$, for which $y_{1}\left(x, \lambda_{m_{1}}{ }^{\prime}\right)$ has $m_{1}$ roots in the interval $a<x<c$ while $y_{1}\left(c, \lambda_{m_{1}}{ }^{\prime}\right)=0$. There is also one and only one value of $\lambda$ in the interval $L>\lambda>l$, say $\lambda_{m_{2}}{ }^{\prime \prime}$, for which $y_{2}\left(x, \lambda_{m_{2}}{ }^{\prime \prime}\right)$ has $m_{4}$ roots in the interval $c<x<b$ while $y_{2}\left(c, \lambda_{m_{2}}{ }^{\prime \prime}\right)=0$. Let us consider the two sequences $\lambda_{0}{ }^{\prime}, \lambda_{1}{ }^{\prime}, \lambda_{2}{ }^{\prime}, \cdots$, and $\lambda_{0}{ }^{\prime \prime}, \lambda_{1}{ }^{\prime \prime}, \lambda_{2}{ }^{\prime \prime}, \cdots$. Each of these sequences is arranged in order of decreasing magnitude. Let us now arrange the terms of the two sequences in a single sequence $\lambda_{0}, \lambda_{1}, \lambda_{2}, \cdots$, in order of decreasing magnitude ; it being understood that if $\lambda_{i}^{\prime}=\lambda_{j}^{\prime \prime}$ these two terms shall be represented by only one term in the new sequence. Let us denote by $\mu_{k}$ the aggregate number of roots of $y_{1}\left(x, \lambda_{k}\right)$ and $y_{2}\left(x, \lambda_{k}\right)$ in $a b$. Then it is clear that in general $\mu_{k+1}=\mu_{k}+1$, the only exception being that if $y_{1}\left(c, \lambda_{k}\right)=y_{2}\left(c, \lambda_{k}\right)=0, \mu_{k+1}=\mu_{k}+2$. In the sequence of integers $\mu_{0}, \mu_{1}, \cdots$, one at least of the two integers $m$ and $m-1$ must, therefore, be present. Let us

$\dagger$ 'This inequality need not of cousse hold for the points of discontinuity of $\phi$. Moreover, there must be some points (besides these poinst of discontinuity; where the equality sign dot's not hold. 
give to $k$ the value which makes $\mu_{k}=m$ if this is possible, and if not the value which makes $\mu_{k}=m-1$.

One case we can dispose of at once, viz., that in which $\mu_{k}=m$ and $y_{1}\left(c, \lambda_{k}\right)=y_{2}\left(c, \lambda_{k}\right)=0$. When $\lambda>\lambda_{k}$ the aggregate number of roots of $y_{1}$ and $y_{2}$ in $a b$ is less than $m$, when $\lambda<\lambda_{k}$ it is greater than $m$. Accordingly when $\lambda=\lambda_{k}$ and for this value only is the aggregate equal to $m$. Moreover, for this value $y_{1}$ and $y_{2}$ are linearly dependent since they both vanish when $x=c$. In this case then our theorem is proved.

Apart from this case, which we now exclude, it is clear that $\lambda=\lambda_{k}$ is not the value of $\lambda$ we are seeking. Leaving aside this value then we see that the aggregate number of roots of $y_{1}$ and $y_{2}$ in $a b$ is equal to $m$ when and only when $\lambda_{k}>\lambda>\lambda_{k+1}$. It remains then to prove that in this range of values of $\lambda$ there is one and only one value for which $y_{1}$ and $y_{2}$ are linearly dependent, $i$. e. (since $y_{1}(c, \lambda) \neq 0$ and $\left.y_{2}(c, \lambda)+0\right)$ for which $y_{1}^{\prime}(c, \lambda) / y_{1}(c, \lambda)=y_{2}^{\prime}(c, \lambda) / y_{2}(c, \lambda)$. This follows at once from Th. of Osc. 1 , theorem II for as $\lambda$ decreases from $\lambda_{k}$ to $\lambda_{k+1} y_{1}^{\prime}(c, \lambda) / y_{1}(c, \lambda)$ will be decreasing continuously while $y_{2}^{\prime}(c, \lambda) / y_{2}(c, \lambda)$ is increasing continuously. Moreover we must have either $y_{1}\left(c, \lambda_{k}\right)=0$ or $y_{2}\left(c, \lambda_{k}\right)=0$ and either $y_{1}\left(c, \lambda_{k+1}\right)=0$ or $y_{2}\left(c, \lambda_{k+1}\right)=0$. Accordingly when $\lambda=\lambda_{k}$ and also when $\lambda=\lambda_{k+1}$ one of the two ratios $y^{\prime} / y$ just considered is infinite. There are four possibilities here, viz.: 1st, $y_{1}^{\prime} / y_{1}$ decreases from $+\infty$ to $-\infty$; $2 \mathrm{~d}, y_{1}^{\prime} / y_{1}$ decreases from $+\infty$ to a finite limit while $y_{2}^{\prime} / y_{2}$ increases from a finite limit to $+\infty ; 3 \mathrm{~d} y_{1}^{\prime} / y_{1}$, decreases from a finite limit to $-\infty$ while $y_{2}^{\prime} / y_{2}$ increases from $-\infty$ to a finite limit; 4th, $y_{2}^{\prime} / y_{2}$ increases from $-\infty$ to $+\infty$. In any case, however, it is clear that the two ratios become equal once and only once, as was to be proved.

In this proof we have tacitly assumed that $m \neq 0$. The changes necessary in the case $m=0$ are however so slight and so obvious that it does not seem necessary to dwell upon them.*

The generalization of Klein's theorem of oscillation follows now so readily that we merely give the result:

$\mathrm{X}$. Theorem VI still holds if we drop at some or all of the points $a_{i}$ and $b_{i}(i=0,1, \cdots, k)$ the requirements that

$$
\chi \cdot e^{2 \int_{\xi_{i}}^{x} p d x} \text { and } \psi \cdot e^{2 \int_{\xi_{i}}^{x} p d x}
$$

* Were it not for this case $m=0$ we might have followed a slightly simpler method analogous to that used by Picard in a somewhat similar case. Cf. Traité d'Analyse, vol. 3 , p. 122 . 
be functions of the kind described in (A) and merely require that these functions when multiplied by $x-a_{i}$ (or by $x-b_{i}$ ) are functions of the kind deseribed in (A) PROVIDED, that at the points $\alpha_{i}$ and $b_{i}$ just mentioned we require that $\alpha_{i}=0$ and $\beta_{i}=0$.

If we apply this theorem to equation (7) we get

XI. Theorem VII still holds if we allow some or all of the points $a_{i}$ and $b_{i}$ to be singular points with unequal real exponents whose difference is no longer restricted to being numerically less than 1, PROVIDED that at each of these points we require that the corresponding solution $y_{i}$ should be proportional to the fundamental solution corresponding to the greater exponent of this point.

Theorems VII and XI include all the cases for which Klein has discussed the theorem of oscillation in his lithographed lectures: Lineare Differentialgleichungen der zweiten Ordnung, 1894, or for which he has there expressed the opinion, based on analogy with simpler cases, that the theorem is probably true.

We close by noting two slight generalizations :

1st The proof of theorems VII and XI would not be in the least affected if instead of the polynomial

$$
C_{n-2} x^{n-2}+\cdots+C_{0}
$$

which enters equation (7) we had a polynomial of degree greater than $n-2$, in which case the point $x=\infty$ would be an irregular point. The number $k+1$ of intervals $a_{i} b_{i}$ might of course then be correspondingly increased.

$2 d$ Theorems VII and XI still remain true, the proofs not being essentially affected, if several of the points $e_{1}, e_{2}, \cdots, e_{n}$ coincide, provided that none of the intervals $a_{i} b_{i}$ reaches up to such a " multiple" point, which will obviously in general be an irregular point.

[Note added Sept. 14, 1898: It was not until the present paper was in print that I noticed that some of the theorems contained in it had been proved for a particular differential equation and in somewhat restricted form in a paper by Van Vleck "On the Polynomials of Stieltjes" in the June number of the BuLletiN. There is even an analogy of method between the proof of Van Vleck's Theorem IV and that of my Theorem IX. It may therefore be well to state that I was in possession of all the results and methods of the present paper last February (Cf. a remark in the middle of p. 365 Th. of Osc. 2) and was prevented from working them out in detail only by press of other work.]

Harvard University, Cambridge, Mass. 